\title{
Facile Sensor for Heavy Metals Based on Thiol-Capped CdTe Quantum Dot
}

\section{Souad A Elfeky*}

National Institute of Laser Enhanced Science, Cairo University, Giza, Egypt

"Corresponding author: Souad A Elfeky, National Institute of Laser Enhanced Science, Cairo University, Giza, Egypt, Tel: +201110199538; E-mail: dr_souad_elfeky@niles.edu.eg

Received date: February 26, 2018; Accepted date: March 07, 2018; Published date: March 13, 2018

Copyright: () 2018 Elfeky SA. This is an open-access article distributed under the terms of the Creative Commons Attribution License, which permits unrestricted use, distribution, and reproduction in any medium, provided the original author and source are credited.

\begin{abstract}
Background: Semiconductor Quantum Dots (QDs) have gained much attention in different fields of application due to exclusive photochemical reactions. They achieved a big triumph in supramolecular chemistry. A highly sensitive and specific nanosensor was reported in this study using QDs coated with acid for sensing Heavy Metal $(\mathrm{HM})$ ions in water. Few relevant patents to the topic have been reviewed and cited in this article.
\end{abstract}

Methods: The Mercaptopropionic Acid (MPA)-capped CdTe QDs were prepared through a hydrothermal route and characterized by TEM, FTIR, UV/Vis, fluorescence and XRD techniques. Sensing of HM ions was performed in Deionized Water (DW) by titration of their respective salts with CdTe/MPA.

Results: TEM revealed that the QDs size diameter was in the range of $4 \pm 0.2 \mathrm{~nm}$. FTIR, UV/Vis, and fluorescence confirmed the structure and spectroscopic classification of QDs. XRD showed that CdTe/MPA crystals were cubic. Based on the fact that the QDs fluorescence possibly quench by HM ions, an easy process for HM detection was anticipated. The QDs response showed a direct correlation to the concentration of HM. The synthesized QDs had potential for detecting the $\mathrm{HM}$ ions with higher selectivity to Mercury ions $\left(\mathrm{Hg}^{2+}\right)$ in water compared with Lead $\left(\mathrm{Pb}^{2+}\right)$ and Barium $\left(\mathrm{Ba}^{2+}\right)$. The optimum sensor sensitivity was achieved at $\mathrm{pH} 8$.

Conclusion: HM ions quenching CdTe/MPA fluorescence in a concentration-dependent manner. The method presented in this manuscript is novel, economical and convenient for realistic applications.

Keywords: Nanosensors; CdTe; Mercury; Lead; Barium

\section{Abbreviations:}

QDs: Quantum Dots; HM: Heavy Metal; MPA: Mercaptopropionic Acid; MAA: Mercaptoacetic Acid; NCs: Nanocrystals; DW: Deionized Water; FTIR: Fourier Transform Infrared.

\section{Introduction}

In the current time, fluorescent QDs have been used in different applications because of their amazing optical properties they are better than organic dyes [1]. QDs Nanocrystals (NCs), turn out to be essential in the industrial field due to their exclusive electronic and spectroscopic characteristics. They achieved great success in supramolecular chemistry [2]. QDs get their importance from the quantum confinement of the excited electrons and holes. This confinement gives rise to unique electronic and optical characteristics that are different from those in bulk semiconductors [3]. Moreover, surface modification of the QDs can enhance their optical properties [4] by obstructing non-radiative electron/hole $\left(\mathrm{e}^{-} / \mathrm{h}^{+}\right)$recombination [5]. The photostability of QDs can be improved by increasing traps sites on the surface. The generation of new traps on the surface of the QD leads to the appearance of new emission bands. For the previous motives, semiconductor QDs has great achievements in analytical applications [6]. Some literature studied the application of thiolcapped QDs in metal ion sensing [7] and significant success was achieved [8]. Schematic illustration of the effect of HM ions in quenching QD fluorescence is presented in Scheme 1.

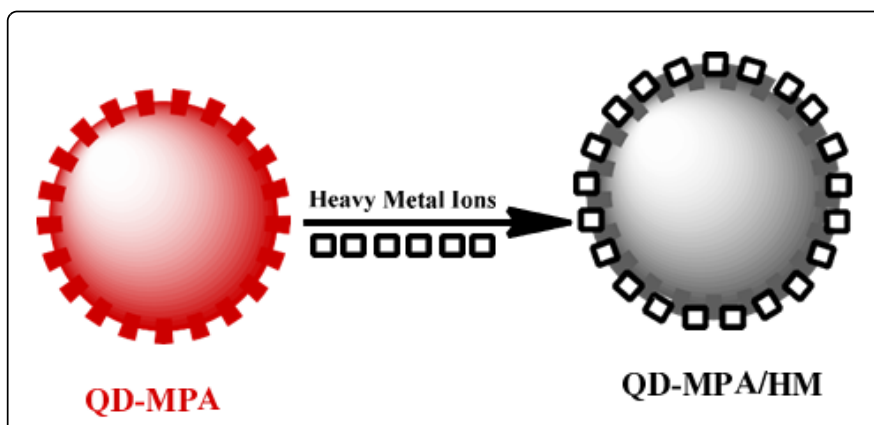

Scheme 1: Quenching of QDs fluorescence upon HM ion complexation.

For instance, Mercaptoacetic Acid (MAA) capped CdS QDs used for Mercury(II) sensing [9], thioglycolic acid capped CdTe QDs applied to Lead(II) sensing [10]. CdTe QDs was applied before for selective sensing of $\mathrm{Cu}^{2+}[11]$. A great interest is paid towards sensitive sensors of analytes that have an environmental impact. Monitoring HMs in aqueous media has great significance due to their dangerous effect on the ecosystem. 
Mercury is well known for its toxic environmental impact. Mercury comes up from natural or industrial sources. It damages microorganisms and aquatic environment at low concentrations. Consuming water polluted with Mercury causes serious disorders in addition to neurological damage [12]. Consequently, developing good sensitive and selective fluorescent sensor to identify the Mercury ion at low concentration in the aqueous medium is of great interest.

Lead ion $\left(\mathrm{Pb}^{2+}\right)$, causes a severe damage to human health and the environment [13]. It is found in the environment and its toxicity to animals comes from its non-degradable nature. Lead-poisoning results in severe neurotoxic effects mainly in children. Inductively coupled plasma mass spectrometry is the conventional method used in Lead detection [14]. This is expensive method and requires complicated sample preparation. Therefore, developing a sensitive sensor for detection of $\mathrm{Pb}^{2+}$ in the environment has a great significance.

Barium is a trace element and cannot be found alone in the environment [15]. Some compounds which occur in nature such as Barium sulfate and Barium carbonate contain Barium. Barium at high doses excites arterial muscle and causes vasoconstriction [16]. Barium can cause death, the time between Barium consumption and death toxicity depend on the quantity and the solubility of the Barium compound. Thus death can happen in a few hours or a few days. The lethal oral dose is between 3 and $4 \mathrm{~g}$ [17].

Only a few workers have reported the presence of Mercury(II) [18] and Lead(II) ions using semiconductor QDs [18]. However, these methods require complex reaction condition, in addition to the high cost and low yield where no work reported the sensing Barium(II) using semiconductor QDs. This study illustrates a facile preparation of the MPA capped CdTe QDs for application in sensing $\mathrm{Hg}(\mathrm{II}), \mathrm{Pb}(\mathrm{II})$ and $\mathrm{Ba}(\mathrm{II})$. This method is straightforward, responsive and economic and can be applied at room temperature conditions.

\section{Materials and Methods}

All chemicals were purchased from Sigma Aldrich-UK.

\section{Synthesis of CdTe/MAA NCs}

MPA-capped CdTe QDs were synthesized in DW according to the procedure described by Zheng [19] with slight modifications. In brief, NaHTe solution, prepared by adding Te and $\mathrm{NaBH}_{4}$ powder to $\mathrm{CdCl}_{2}$ solution under an inert condition in the presence of MPA. $\mathrm{Cd}^{2+}: \mathrm{Te}^{2-}: \mathrm{MPA}$ molar ratio was 1.0:0.5:2.0. Then, $\mathrm{pH}$ of the solution was adjusted to 9.0-9.5 using $1 \mathrm{M} \mathrm{NaOH}$ solution. After that, the reaction mixture was heated $\left(100^{\circ} \mathrm{C}\right)$ and refluxed to get MPA-capped CdTe QDs. Absolute ethanol was added to separate the MPA-capped CdTe QDs. This process was (precipitation by ethanol) repeated for three cycles to purify the product.

\section{Characterization}

- Absorption spectra were recorded using a Perkin-Elmer Lambda 20 spectrometer.

- FTIR spectra recorded with FT/IR-4600 spectrometer (JASCO, Japan).

- CdTe/MPA crystal structure was studied using the X-ray diffractometer system X'Pert ${ }^{3}$ Powder (PANalytical, The Netherlands).

- Fluorescence spectra were recorded with the Perkin Elmer LS 55 fluorescence spectrometer
- Microscopic imaging was acquired by JOEL USA transmission electron microscope.

\section{Sensing of HM ions}

The luminescent MPA-capped QDs were dissolved in DW; concentration of QDs is $1.0 \mathrm{mg} \mathrm{L}^{-1}$ [16]. The stock solutions of metal ions were prepared by dissolving their respective salts $\left(\mathrm{HgCl}_{2}, \mathrm{PbCl}_{2}\right.$, $\mathrm{BaCl}_{2}$ ) in $\mathrm{DW}$, the used concentration of $\mathrm{Hg}^{2+}, \mathrm{Pb}^{2+}$, and $\mathrm{Ba}^{2+}$ ions is ranging from 0.4-3.6 ppm. A stock solution of $244 \mu \mathrm{M}$ from each $\mathrm{HM}$ was prepared in DW and $5 \mu \mathrm{L}$ was titrated (9 times) to $3 \mathrm{ml}$ of MPA/ CdTe $\left(1 \mathrm{mgL}^{-1}\right)$ in DW. In $\mathrm{pH}$ effect experiment, $\mathrm{HCl}(1 \mathrm{~N})$ and $\mathrm{NaOH}$ $(1 \mathrm{M})$ were used to adjust the $\mathrm{pH}$ to the proposed value using JENWAY $3505 \mathrm{pH}$ meter. Origin 8 software was used for data processing, statistical analysis, and $\mathrm{K}_{\mathrm{SV}}$ calculations.

\section{Results and Discussion}

\section{Characterization of MPA-capped CdTe QDs}

Fourier Transform Infrared (FTIR) spectrometry is a great tool to describe the atoms on the surface. FTIR spectrometry is the unsurpassed technique to chase the surface modification of the synthesized chemical species [20].

Figure 1 shows the FTIR spectra of (a) free MPA and (b) MPAcapped CdTe QDs. The IR absorption bands at $3034 \mathrm{~cm}^{-1}(\mathrm{vOH}$, $\mathrm{COOH}), 2550-2650 \mathrm{~cm}^{-1}(v \mathrm{~S}-\mathrm{H}), 1710 \mathrm{~cm}^{-1}(v \mathrm{C}=\mathrm{O}), 1400 \mathrm{~cm}^{-1}$ $(v \mathrm{COOH})$, and $1277 \mathrm{~cm}^{-1}(v \mathrm{C}-\mathrm{O})$ are for MPA. The bands at $3410 \mathrm{~cm}$ ${ }^{-1}$ ( $(\mathrm{OH}, \mathrm{COOH}), 1380-1555 \mathrm{~cm}^{-1}$ (vs $\left.\mathrm{COOH}\right)$ and $1290 \mathrm{~cm}^{-1}$ (vCO) are for MPA-capped CdTe QDs. MPA-capped CdTe QDs and free MPA FTIR illustrated the presence of carboxyl and carbonyl groups absorption peaks. This confirms the coexistence of these groups on the shell of the CdTe QDs. The missing of S-H $\left(2550-2650 \mathrm{~cm}^{-1}\right)$ vibration peak in MPA-capped CdTe QDs may be due to the covalent bonding between thiols and Cd atom on the QDs surface. These results agree with Zhang [21] who found that the sulfhydryl group band at $2567 \mathrm{~cm}^{-1}$ lost in the FTIR spectrum of MPA-capped QDs.

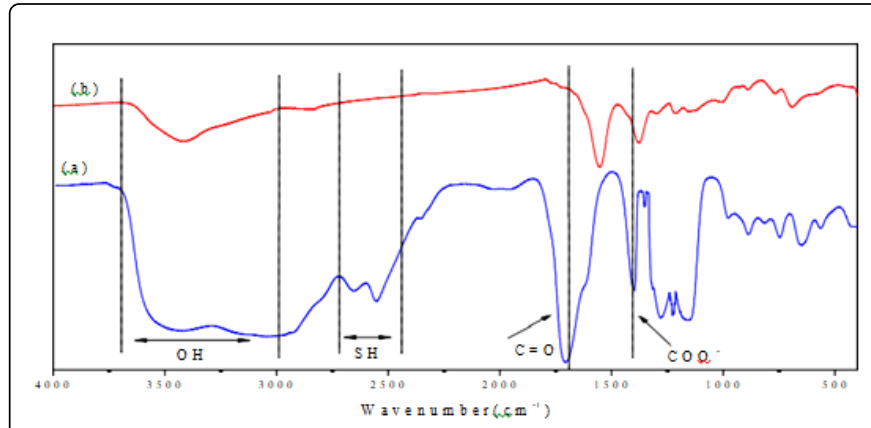

Figure 1: FTIR spectrum of (a) MPA (b) MPA-capped CdTe QDs.

The morphology and size of the prepared QDs were studied by the TEM. The image in Figure 2a shows that the MPA-capped QDs are dispersed and mostly spherical particles. HRTEM in Figure $2 \mathrm{~b}$ reveals that the average crystal size is $4 \pm 0.2 \mathrm{~nm}$.

The XRD wide diffraction peak at $2 \theta=26.7$ of the prepared CdTe QDs (Figure 2c) can be indexed to the (200) reflection for cubic CdTe complying with the standard pattern (JCPDS card 75- 2083). The 
broad peak indicates the occurrence of small size nanoparticles $[22,23]$.

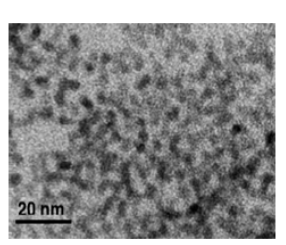

(a)

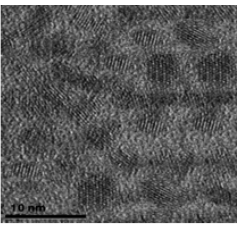

(b)

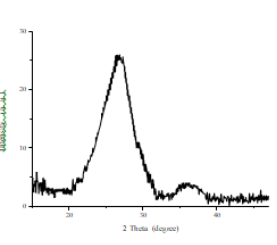

(c)
Figure 2: (a) TEM, (b) HRTEM (c) XRD of MPA/CdTe QDs.

From the XRD data, the diameter of the MPA/CdTe QDs can be calculated using Scherrer formula [24] as shown in equation (1):

\section{$\mathrm{d}=\mathrm{K} \lambda / \beta \cos \theta(1)$}

Where, $\mathrm{d}=$ crystal size, $\mathrm{K}=$ Scherrer constant $(0.89), \lambda=$ wavelength of the $\mathrm{X}$-ray radiation $(0.15418 \mathrm{~nm}$ for $\mathrm{Cu} \mathrm{Ka})$ and $\beta=$ full width at half maximum of diffraction peaks measured at $2 \theta$.

From equation (1), the calculated QDs crystal size is $3.5 \mathrm{~nm}$. The calculated size usually $10-15 \%$ less than the size of particles that appears under electron microscopy as the X-ray coherent scattering applies to the internal side of the crystal and does not include margins.

The absorption and emission of MPA-capped CdTe QDs were measured by UV-visible absorption spectrometry and fluorescence spectroscopy as shown in Figure 3. The absorption peak of the MPAcapped CdTe nanoparticles is noticed at $520 \mathrm{~nm}$ and the fluorescence emission is found at $545 \mathrm{~nm}\left(\lambda_{\mathrm{ex}}=400 \mathrm{~nm}\right)$. In fact CdTe NCs generally have no luminescent properties until they are stabilized with mercapto compounds as mentioned by Wei-Heng in his patent entitled: Water soluble nanocrystalline QDs [25].

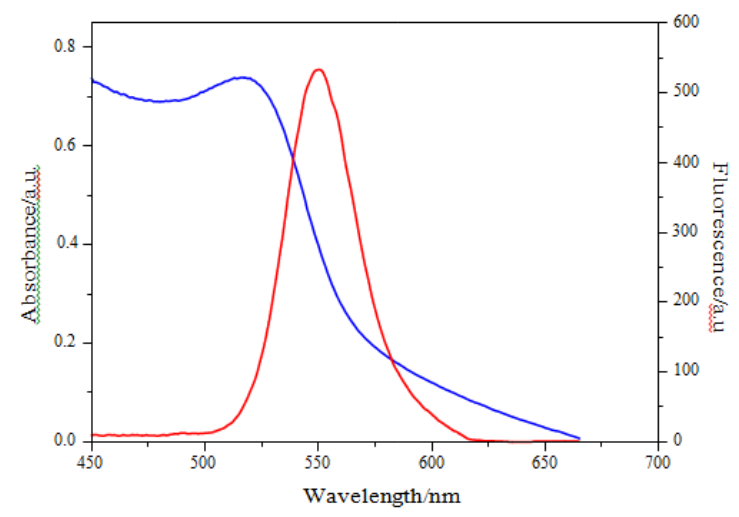

Figure 3: Absorption and fluorescence spectrum of CdTe QDs and MPA-capped CdTe QDs.

\section{Effect of $\mathrm{pH}$ on the CdTe/MPA sensitivity to HM}

The $\mathrm{pH}$ has a significant effect on the fluorescence intensity of the system. In addition, the $\mathrm{pH}$ affects the solubility of the QDs and its interaction in the system. Kotov [26] mentioned in his patent that $\mathrm{CdTe}$ luminescence displays strong sensitivity to the $\mathrm{pH}$ of the media.
$\mathrm{He}$ also explained that the variation of $\mathrm{pH}$ will change the electrostatic attraction between CdTe and metals. The $\mathrm{pH}$ in the range between 4 and 11 was studied to select the optimum sensitivity $\mathrm{pH}$. From Figure 4 , it is obvious that maximum sensitivity of CdTe/MPA was at $\mathrm{pH} 8$ for all the tested HM ions.

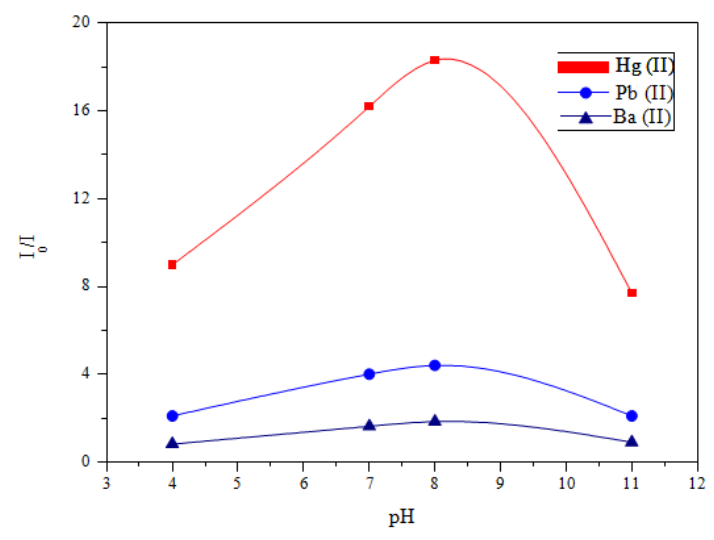

Figure 4: Normalized fluorescence of MPA-capped CdTe QDs $\left(\lambda_{\max }\right.$ $546.4 \mathrm{~nm})$ at different $\mathrm{pH}$ values, HM Concentration $=3.6 \mathrm{ppm}$.

The previous study also confirmed that the optimum sensitivity of QDs to HM was at $\mathrm{pH} 8$ [16]. The QDs sensitivity decrease in the acidic medium may be due to the dissociation of $\mathrm{Cd}^{2+}-\mathrm{MPA}-\mathrm{QDs}$ as the surface binding thiolates protonated in acidic medium [27]. The fluorescence intensity enhanced with increasing the $\mathrm{pH}$ increase up to 8. This can be ascribed to the deprotonation of MPA thiol group at higher $\mathrm{pH}$, as a consequence, the covalent bond between thiol and $\mathrm{Cd}$ atom at the surface of QDs becomes stronger. Thus, the increase of $\mathrm{pH}$ maintains carboxylic acid group negative charge and promotes the interaction with $\mathrm{HM}$ ions [28]. At $\mathrm{pH}>8$, the fluorescence intensity decreases due to hydration of the QD surface. Furthermore, hydroxides of $\mathrm{HM}$ ions are precipitated at high $\mathrm{pH}$ values.

The fluorescence response of MPA-capped CdTe QDs to different $\mathrm{Hg}^{2+}, \mathrm{Ba}^{2+}$ and $\mathrm{Pb}^{2+}$ concentrations at $\mathrm{pH} 8$ is illustrated in Figure 5. Figure 5 displays the fluorescence emission spectra of MPA-capped CdTe QDs containing various concentrations of (a) $\mathrm{Hg}^{2+}$, (b) $\mathrm{Ba}^{2+}$ and (c) $\mathrm{Pb}^{2+}$ respectively. Fluorescence intensity was regularly quenched by adding more HMs, this may be due to the change on the surface of QDs after HM addition. Fluorescence quenching of CdTe QDs as a response to $\mathrm{HM}$ addition can be explained in view of electron transfer from MPA to $\mathrm{Hg}^{2+}, \mathrm{Ba}^{2+}$ or $\mathrm{Pb}^{2+}$ [29]. The QD sensing to $\mathrm{HM}$ was patented before by $\mathrm{Wu}[30]$ who found that QD fluorescence emission energy transfer can detect the presence and the quantity of the HM. 


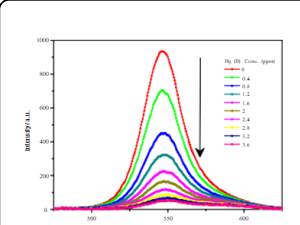

(a)

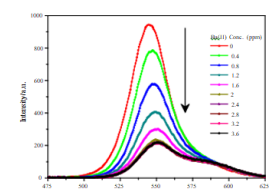

(b)

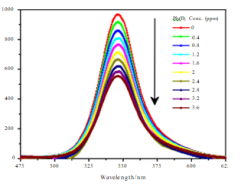

(c)
Figure 5: MPA-capped CdTe QDs (QDs $\left(1 \mathrm{mg} \mathrm{L}^{-1}, \lambda_{\mathrm{ex}}=400 \mathrm{~nm}\right.$ ) fluorescence quenching in the presence of $\mathrm{Hg}^{2+}(\mathrm{a}), \mathrm{Ba}^{2+}(\mathrm{b})$ and $\mathrm{Pb}^{2+}(\mathrm{c})$ at various concentrations and $\mathrm{pH} 8$.

Comparing the QDs sensing results of different HMs, it was found that the used HMs quench the fluorescence of QDs in a concentrationdependent manner. Similar results were obtained by Ming [31] who found that L-cysteine modified CdTe QD fluorescence intensity changes in relationships with Mercury concentration. In this study, when increasing the concentrations of $\mathrm{Hg}^{2+}, \mathrm{Ba}^{2+}$ and $\mathrm{Pb}^{2+}$, the fluorescence intensity MPA-capped QDs gradually reduced to 4\%, 22\% and $54 \%$ (respectively) of the initial intensity after the primary sharp decline, while the fluorescence of $\mathrm{Ba}^{2+}$ treated QDs continues on decreasing till getting the plateau. Numerous quenching approaches have been anticipated to describe QDs fluorescence quenching by metal ions. Some mechanisms such as inner filter effect, ion binding interaction, electron transfer process, and non-radiative recombination pathway are capable of explaining the quenching phenomena $[32,33]$. From the results, there was no obvious shift in emission wavelength (emission band centered $545 \mathrm{~nm}$ with the excitation $400 \mathrm{~nm}$ ) by adding more concentration from HM ions. Thus, it is suggested that the quenching in this system is ascribed to enhancing non-radiative recombination of excited electrons $\left(\mathrm{e}^{-}\right)$and holes through the effective electron transfer from MPA to HM ions [34].

It was reported that ion sensitivity and selectivity depend on the function groups of the QDs surface [34]. It is known that mercapto acids molecules have high tendency as chelating agents for $\mathrm{Hg}^{2+}$ than other HM ions [35]. In our study, the ion sensitivity was in the following order $\mathrm{Hg}(\mathrm{II})>\mathrm{Ba}(\mathrm{II})>\mathrm{Pb}(\mathrm{II})$. A patent entitled "Sensors using AIGaN/GaN high electron mobility transistors" invented by Fan [35], also used thioglycolic acid as a chelating ligand and sensor binding site for HM detection in solution. From the results, it is obvious that QDs fluorescence quenching boosted by increasing HM concentration that can be represented by the Stern-Volmer relationship which is given by the following equation (2):

$$
\mathrm{I}_{0} / \mathrm{I}=1+\mathrm{K}_{\mathrm{SV}}[\mathrm{HM}](2)
$$

$\mathrm{I}_{0} / \mathrm{I}=$ fluorescence intensities of QDs in the absence and presence of $\mathrm{HM}$, respectively. $\mathrm{HM}=$ heavy metal concentration, $\mathrm{K}_{\mathrm{SV}}=$ Stern-Volmer constant [36].

The slope of the $\mathrm{I}_{0} / \mathrm{I}$ versus the concentration curve (Figure 6 ) is the Stern-Volmer constant $\mathrm{K}_{\mathrm{SV}}$ [37]. Table 1 illustrates the $\mathrm{K}_{\mathrm{SV}}$, Standard Error (SE) and R-square $\left(\mathrm{R}^{2}\right)$ values for CdTe/MPA QDs interaction with the used HM.

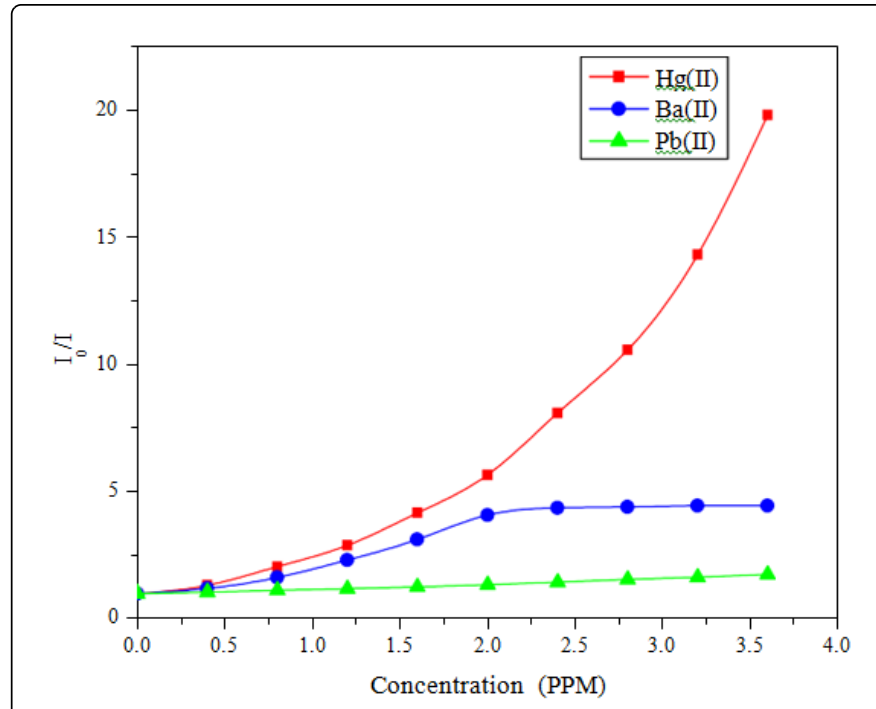

Figure 6: Stern-Volmer plot for the quenching of CdTe/MPA QDs $\left(1.0 \mathrm{mg} \mathrm{L}^{-1}\right)$ by $\mathrm{HM}$ at $\lambda_{\max } 546.4 \mathrm{~nm}$ and $\mathrm{pH} 8$.

\begin{tabular}{|l|l|l|l|}
\hline $\mathbf{H M}$ & $\mathbf{K}_{\text {SV }}$ & SE & $\mathbf{R}^{2}$ \\
\hline $\mathrm{Hg}(\mathrm{II})$ & 23.1 & 2.4 & 0.9 \\
\hline $\mathrm{Ba}(\mathrm{II})$ & 4.5 & 0.58 & 0.85 \\
\hline $\mathrm{Pb}(\mathrm{II})$ & 0.96 & 0.03 & 0.99 \\
\hline
\end{tabular}

Table 1: $\mathrm{K}_{\mathrm{SV}}, \mathrm{SE}$ and $\mathrm{R}^{2}$ values for CdTe/MPA QDs interaction with $\mathrm{Hg}$ (II), $\mathrm{Ba}(\mathrm{II})$ and $\mathrm{Pb}$ (II).

\section{Conclusion}

The HM ions quench the fluorescence of the MPA-capped CdTe QDs in a concentration-dependent manner. The quenching was found to be relative to the amount of HM ions that can be described by the Stern-Volmer relationship. The MPA-capped CdTe shows a higher affinity for $\mathrm{Hg}^{2+}$ than other used $\mathrm{HM}$ ions. The optimum sensor sensitivity was achieved at $\mathrm{pH} 8$.

\section{Acknowledgement}

The author is very grateful to the University of Cairo and University of Bath (Chemistry Department) for supporting this research.

\section{Conflict of Interest}

The author declares that there is no conflict of interest regarding the publication of this paper.

\section{References}

1. Rosenthal SJ, Chang JC, Kovtun O, McBride JR, Tomlinson ID (2011) Biocompatible quantum dots for biological applications. Chem Biol 18: 10-24.

2. Aguilera-Sigalat J, Casas-Solvas MJ, Morant-Minana CM, VargasBerenguel A, Raquel EG, et al. (2012) Quantum dot/cyclodextrin supramolecular systems based on efficient molecular recognition and their use for sensing. Chem Commun 48: 2573-2575. 
3. Andrew MS, Shuming N (2010) Semiconductor Nanocrystals: Structure, Properties, and Band Gap Engineering. Acc Chem Res 43: 190-200.

4. Vidhya K, Saravanan M, Bhoopathi G, Devarajan PV, Subanya S (2014) Structural and optical characterization of pure and starch-capped $\mathrm{ZnO}$ quantum dots and their photocatalytic activity. Appl Nanosci 5: 235-243.

5. Andrew MS, Aaron MM, Shuming N (2009) Tuning the optical and electronic properties of colloidal nanocrystals by lattice strain. Nat Nanotechnol 4: 56-63.

6. Fernandez-Argueelles MT, Jin WJ, Costa-Fernandez JM, Pereiro R, SanzMedel A (2005) Surface-modified CdSe quantum dots for the sensitive and selective determination of $\mathrm{Cu}$ (II) in aqueous solutions by luminescent measurements. Anal Chim Acta 549: 20-25.

7. Chen FY, Rosenzweig Z (2002) Luminescent CdS Quantum Dots as Selective Ion Probes. Anal Chem 74: 5132-5138.

8. $\quad$ Ge S, Zhang C, Zhu Y, Yu J, Zhang S (2010) BSA activated CdTe quantum dot nanosensor for antimony ion detection. Analyst 135: 111-115.

9. Masilamany K, Ramaier N (2009) Mercaptoacetic acid capped CdS quantum dots as fluorescence single shot probe for Mercury(II). Sensors Actuat B-chem 139: 91-96.

10. Haiyan W, Qiongfang C, Zhian T, Xunxun Y, Lun W (2012) Electrochemiluminescence of $\mathrm{CdTe}$ quantum dots capped with glutathione and thioglycolic acid and its sensing of $\mathrm{Pb} 2+$. Electrochem Acta 72: 28-31.

11. Zhang L, Shang L, Dong S (2008) Sensitive and selective determination of $\mathrm{Cu} 2+$ by electrochemiluminescence of CdTe quantum dots. Electrochem Commun 10: 1452-1454.

12. Clarkson TW, Magos L, Myers GJ (2003) The Toxicology of Mercury Current Exposures and Clinical Manifestations. N Engl J Med 349: 1731-1737.

13. Flegal AR, Smith DR (1995) Measurements of Environmental Lead Contamination and Human Exposure. Rev Environ Contam Toxicol 143: 1-45.

14. Ali EM, Zheng YG, Yu HH, Ying JY (2007) Ultrasensitive Pb2+ Detection by Glutathione-Capped Quantum Dots. Anal Chem 79: 9452-9458.

15. US Environmental Protection Agency, Office of Drinking Water. US EPA (1985) Health advisory - barium. Washington, DC.

16. Stockinger HE (1981) The metals. In: Clayton GD, Clayton FE (eds.). Patty's industrial hygiene and toxicology. 3rd edn. John Wiley, New York, USA 2: 1493-2060.

17. Reeves AL (1986) Barium. In: Friberg L, Nordberg GF, Vouk VB (eds.) Handbook on the toxicology of metals. 2nd edn. Amsterdam, Elsevier/ North Holland Biomedical Press, pp. 84-94.

18. Xia YS, Zhu CQ (2008) Use of surface-modified CdTe quantum dots as fluorescent probes in sensing Mercury(II). Talanta 75: 215-221.

19. Zheng L, Wanga Y, Zhang G, Xu W, Han Y (2010) Chemiluminescence resonance energy transfer in the luminol CdTe quantum dots conjugates. J Lumine 130: 995-999.

20. Baraton MI (1998) The Surface Characterization of Nanosized Powders: Relevance of the FTIR Surface Spectrometry. Nanostruct Mat 50: 303-317.

21. Zhang BH, Wu FY, Wu YM, Zhan XS (2010) Fluorescent Method for the Determination of Sulfide Anion with Zns:Mn Quantum Dots. J Fluoresc 20: $243-250$
22. Haifeng B, Na H, Yang Y, Dongyuan Z (2010) Biosynthesis of biocompatible cadmium telluride quantum dots using Yeast cells. Nano Res 3: 481-489.

23. Monras PJ, Diaz V, Bravo D, Montes RA, Chasteen TG, et al. (2012) Enhanced Glutathione Content Allows the In Vivo Synthesis of Fluorescent Cdte Nanoparticles by Escherichia Coli. PLOS ONE 7: 48657.

24. Azaoff LV (1968) Elements of X-ray Crystallography. McGraw-Hill Book Co., New York.

25. Wei-Heng S, Wan YS, Li H, Schillo CM (2011) Water soluble nanocrystalline quantum dots. US20110001092 A1.

26. Kotov N, Lee J, Govorov A (2011) Nanoparticle assemblies with molecular springs. Angewandte Chemie International Edition 44: 7439-7442.

27. Gao M, Kirstein S, Mohwald H, Rogach LA, Kornowski A, et al. (1998) Strongly Photoluminescent CdTe Nanocrystals by Proper Surface Modification. J Phys Chem B 102: 8360-8363.

28. Landes C, Burda C, Braun M, El-Sayed AM (2001) Photoluminescence of CdSe Nanoparticles in the Presence of a Hole Acceptor: n-Butylamine. J Phys Chem B 105: 2981-2986.

29. Ting-Ting G, Yu-Jun Z, Nan-Jing Z, Xue X, Gao-Fang Y, et al. (2012) Hydrothermal synthetic mercaptopropionic acid stabled CdTe quantum dots as fluorescent probes for detection of Ag+. Spectrochim Acta A 99: 62-68.

30. Wu N, Xiaodong S, Lawrence H (2009) Quantum dot-DNA-metallic nanoparticle ensemble as fluorescent nanosensor system for multiplexed detection of heavy metals. US 2009/0200486 A1.

31. Ming S, Ling Z, Bingtai C, Daoliang L, Yaoguang W, et al. (2015) Apparatus and method for detecting Mercury in water body based on quantum dot fluorescent probe [Machine Translation]. CN 105067575 A 20151118.

32. Li BH, Qu GF (2007) Synthesis of Cdte Quantum Dots in Sol-GelDerived Composite Silica Spheres Coated with Calix[4]Arene as Luminescent Probes for Pesticides. Chem Matter 19: 4148-4154.

33. Soliman EM, Mahmoud ME, Ahmed SA (2010) Reactivity of the Thioglycolic Acid Physically and Chemically Bound to Silica Gel as New Selective Solid Phase Extractor for Removal of Heavy Metal Ions From Natural Water Samples. Intern J Environ Anal Chem 82: 403-413.

34. Vázquez-González M, Carrillo-Carrion C (2014) Analytical strategies based on quantum dots for heavy metal ions detection. J Biomed Opt Vol 19.

35. Fan R, Stephen JP, Tanmay L, Hung-Ta W, Byoung-Sam K (2009) Sensors using AIGaN/GaN high electron mobility transistors. Vol. 94 WO2009039298 A2

36. El-Daly SA, Salem IA, Hussein MA, Asiri AM (2015) Fluorescence Quenching N,N-Bis(2,6-Dimethylphenyl)-3,4:9,10 Perylenetetracarboxylic Diimide (BDPD) Laser Dye by Colloidal Silver Nanoparticles. J Fluoresc 25: 379-385.

37. Lakowicz JR (2013) Principles of Fluorescence Spectroscopy. Springer. 